\title{
Secondary Metastatic Gastric Melanoma
}

\author{
AAM CHOWDHURY ${ }^{\mathrm{a}}, \mathrm{MU}^{\mathrm{K} H A N^{\mathrm{b}}}$, MM HUSSAIN $^{\mathrm{c}}$
}

\begin{abstract}
:
Gastrointestinal melanomas are commonly metastatic from a cutaneous origin. They cause significant cancer related mortality.
\end{abstract}

A 47-year - old male patient presented with severe pallor and prostration due to profuse upper GI bleeding. He was urgently managed with fluid and blood transfusion. Endoscopy revealed gastric mass and $C T$ showed gastric neoplasm with abdominal lymphadenopathy but endoscopic biopsy revealed inflammatory lesion. He had history of malignant melanoma of right third toe three years back which

\section{Introduction:}

Malignant melanomas are tumors of melanocyte derived from neuro-ectoderm and distributed to integument, oropharynx, eyes, meninges and anus. As originated from ectodermal tissue they should be called 'melanocarcinoma' but historically they were termed Malignant Melanoma ${ }^{1}$. They are slow but aggressive tumours causing lymphatic dissemination and less sensitivity to traditional cancer therapies. American cancer society estimates that $1,00,350$ new cases of malignant melanoma will be diagnosed and 6,850 patients will die in US in $2020^{2}$. Gastrointestinal melanomas are usually metastatic and primary gastrointestinal melanomas are very rare finding. As normal gastric epithelium has no

a. Dr Abdullah Al Mamun Chowdhury, FCPS, Junior Consultant (Surgery), Rangamati General Hospital, Rangamati

b. Dr Meshkat Uddin Khan, FCPS, Assistant Professor, Department of Surgery, Chattogram International Medical College, Chattogram

c. Prof. Dr Md. Margub Hussain, FCPS, FICS, MHPEd, Retired Professor of Surgery, DMC\&H

Adddress of Correspondence: Dr Abdullah Al Mamun Chowdhury, FCPS, Junior Consultant (Surgery), Rangamati General Hospital, Rangamati, E-mail: aamc235@gmail.com, Contact no: 01716566299

Received: 25 August, 2020

Accepted: 06 October, 2020 was managed with surgery and chemotherapy. Exploratory laparotomy was done and biopsy was taken from gastric lesion as well as enlarged lymph nodes. Histopathology revealed melanoma from both specimens.

Prognosis is bad. Counseling and collaboration with different relevant disciplines are essential. Management is directed to symptom relieve as well as lengthening survival.

Keywords: Melanoma, Gastric Melanoma

(J Bangladesh Coll Phys Surg 2021; 39: 68-75)

DOI: https://doi.org/10.3329/jbcps.v39i1.50463z

melanocyte, metastatic spread is the first hypothesis if found. Ectopic migration of melanocyte precursors or differentiations of the APUD cells to melanocyte are the possibilities of developing malignant melanoma in stomach ${ }^{3}$. Here we discussing a case of metastatic gastric melanoma presented to us with profuse upper GI bleeding and severe anemia.

\section{Case report:}

A 47 year old male was admitted to a private hospital with the history of multiple episodes of hematemesis and melena for two days.

The patient had a history of malignant melanoma of right third toe three years back. CT scan of inguinal region and pelvis revealed right inguinal lymphadenopathy with no other abnormality. Amputation of the affected toe was performed that time with wide excision of enlarged right inguinal and femoral lymph nodes. Histopathology revealed malignant melanoma for the amputated toe specimen and lymph nodes showed metastatic deposition of malignant melanoma with area of hemorrhage and necrosis. After oncological consultation, chemotherapy with CVD (Cisplatin, Vinblastin, Dacarbazine) regimen was planned for six cycles and completed. Post chemotherapy CT scan revealed multiple hypodense lesions of varying size with nodular enhancement in both lobe of liver as well as 
a nodule in anterior segment of upper lobe of left lung which suggested distant metastasis. Another schedule of chemotherapy with oral Temozolomide was advised as four weekly cycle. After first cycle CT scan revealed disappearance of liver and lung shadows. Five cycle chemotherapy was continued. Next two years was uneventful and he was on routine follow up.

On examination, he was pale, trachycardic but well oriented. Clinically he was severely anaemic, hypotensive, trachycardic with normal abdominal examination findings. Previous operation sites over right foot and groin was healthy. No palpable lymph nodes were found at both inguinal, femoral, external iliac region and no neck glands were palpable. On admission his haemoglobin level was $4.0 \mathrm{mg} / \mathrm{dl}$. His blood group was $\mathrm{A}+\mathrm{ve}$. He was known diabetic and on insulin therapy. Fluid and packed cell transfusion was advised urgently for resuscitation. Endoscopy of upper GI was done. Endoscopy revealed a medium sized round mass lesion with proliferative round wall and central ulcer crater with dark blood clots on posterior wall of lower gastric body. Biopsy was taken and differential diagnosis was suggested between Carcinoma stomach and GIST (gastrointestinal stromal tumour). After transfusion of five units of packed cell his hemoglobin was raised upto $11.2 \mathrm{~g} / \mathrm{dl}$.

We advised a new CT scan of abdomen which revealed a ST density mass in lateral, supero-lateral wall of body and fundus of stomach suggesting carcinoma stomach with upper abdominal lymphadenopathy and two small right hepatic SOL suggestive of metastasis. Histopathology (biopsy taken through upper GI endoscopy) revealed multiple fragments of inflamed and ulcerated gastric mucosa with infiltration of inflammatory cells. No evidence of malignancy is seen.

Discussion with patient and attendants was done in several settings. Pros and cons of different diagnostic as well as management options were clarified. The shortage of newer diagnostic modalities like endoscopic ultrasound and biopsy, PET scan etc. were also described. As a feasible option, exploratory laparotomy was planned with a view of possible palliative gastric resection. With all preoperative evaluation and anesthetic check up patient underwent general anaesthesia and abdomen explored through upper midline incision. There were multiple visible black lymph nodes over greater and lesser omentum. Liver felt normal on palpation. No ascites. Stomach shows multiple indurated areas all over greater curvature upto GE junction. Normal gastric tissue between two induration was very minimum and was not sufficient for any resection and repair or anastomosis. Serosa showed some area of black hue over indurated areas. Per operative discussion between surgeons as well as with patient party was done and procedure directed towards open biopsy from gastric lesion and lymph nodes. Small anterior gastrotomy performed and biopsy taken from ulcerated gastric lesion. Lymph node biopsy was taken from right gastroepiploic nodes. Gastrotomy was closed by two layers, a drain was kept at hepatorenal pouch of Morisson in situ and abdomen closed by layers. On third postoperative day liquid diet started and patient was discharged on fifth with normal oral intake. Histopathology revealed ulcerated lesion of stomach show deposits of melanoma and three out of four lymph nodes show metastatic deposits of malignant melanoma. Sections further requested for immunohistochemistry (Protein S100, HMB-45, Melan A) and consultation with oncologist for further therapy was advised.

Immunohistochemistry from blocks of metastatic lymph nodes suggested metastatic adenocarcinoma originated from gastrointestinal tract. Blocks were advised for further review. Patient is currently admitted to a specialized cancer hospital under supervision of medical oncologist. Next treatment will be chemoradiation for metastatic melanoma.

\section{Discussion:}

Metastatic gastric melanomas are rare but primary gastric melanomas are rarer. Cutaneous melanoma spreads through lymphatic route mainly with a potential to metastasize anywhere. Exception is uveal melanoma- as it cannot spread through lymphatics, it spreads hematogenous ${ }^{4}$. Gastrointestinal metastasis of malignant melanoma occurs 1-4\%. But autopsy reveals opposite data (about $60 \%)^{5}$. GI metastasis of melanoma commonly deposits to jejunum and ileum, followed by colon, rectum and then stomach ${ }^{6}$. One 
case of secondary melanoma in Gall Bladder was reported by Hussainet $\mathrm{al}^{7}$.In practice, diagnosis of primary gastrointestinal melanoma is by exclusion of any primary cutaneous site ${ }^{8}$

Patient with metastatic gastric melanoma presents with vague symptoms (like: nausea, fatigue, dyspepsia etc), mass effect, hematemesis and melena. One small study shows anemia is the most common presentation (about $82 \%$ ) for metastasis of melanoma to GI tract ${ }^{9}$. Usual presentation after few years of treated cutaneous melanoma (median time is 52 months ${ }^{10}$. Diagnosis is delayed except bleeding manifestations.

Endoscopic examination of GI tract, CT scan, MRI, 18-FDG PET scan, histopathology and immunohistochemistry are the modalities for diagnosing gastric melanoma. Classic endoscopic appearance is "polypoid lesions commonly include gastric body and fundus (mainly greater curvature) may show pigmentation and ulceration to produce 'bull's eye' or 'target like' appearance". Amelanotic lesions are possible and difficult to differentiate from gastric adenocarcinoma. There are no characteristic features in endoscopy to confirm malignant gastric melanoma as morphology varies ${ }^{11}$. Digital GI radiography can reveal area of thickened, rigid gastric wall with inability to perform peristaltic movement. CT scan can show a low density mass with or without muscular invasion and lymphadenopathy. Melanoma shows enhancement with contrast at all phase. Melanoma produces melanin which is a paramagnetic substance. On MRI melanin containing melanoma shows hyperintensity on T1 weighted image (T1WI) and diffusion weighted image (DWI) but hypointensity on T2 weighted image $(\mathrm{T} 2 \mathrm{WI})^{12}$. 18 -FDG PET scanning is used to determine the extent of metastasis. It is $92 \%$ sensitive and $100 \%$ specific $^{13}$

Histopathology from endoscopic or open biopsy specimen will show neoplastic proliferation with melanin deposition. Confirmation of melanoma needs immunoreactivity with protein S100, HMB-45 antibody and Melan A. Molecular mechanism includes oncogenes specially BRAF and the microphthalmia-associated transcription factor
(MITF) pathway plays important role in melanoma pathogenesis $^{14}$

As not a common diagnosis, gastric melanoma lacks standard management protocol ${ }^{15}$. Early and solitary metastasis can be dealt with resection but advance cases are difficult to achieve good outcome from surgery. Resections and excision of metastases are usually palliative. Study shows metastasectomy is possible for $33.6 \%$ of the study population and they had improved 5 year survival as well as overall survival compared to patient who cannot ${ }^{16}$. Systemic therapy has low efficacy but practiced widely. Dacarbazine and its active metabolite temozolomide are the most active single agents. Clinical response is good in only $15-20 \%$ of patients and a complete response in $3-5 \%$ of patients and the median duration of response is 4-6 months ${ }^{17}$. USFDA approved biological agent for stage IV melanoma is interleukin-2. Combination of chemotherapeutic agents (i.e. dacarbazine, vinblastin, cisplatin) with biological agent (i.e. interleukin-2, interferon alfa) were tried and known as biochemotherapy but failed to prove any survival benefit. Toxicity supersedes compliance of biochemotherapy ${ }^{18}$. Meta-analysis shows immunotherapy is better than chemotherapy. Combined use of immunotherapy (i.e. ipilimumub, nivolumub) with pembrolizumub should be a better choice due to high efficacy and low toxicity ${ }^{19}$. Historically melanomas are thought to be radio-resistant.

Prognosis for gastric metastasis of melanoma is not good. It indicates stage IV disease and not curable. Study shows median survival ranges 24 to 127 months for nodal disease and 3 to 13.2 months for patient with visceral metastasis ${ }^{20}$. Surgical resection shows some survival benefit when combined with other modalities. Prognostic factors may include young age, less number of sites of metastasis, disease free interval before distant metastasis and resectibility of metastasis ${ }^{21}$

\section{Conclusion:}

Metastatic gastric melanoma is a disease with grim prognosis. Lack of experience and suspicion should not take clinician to a windy path of diagnosis and management. Multidisciplinary approach is 
warranted. Every modality should be tried with hope. Survival benefit means the best palliation for such situation.

\section{Conflict of interest and consent:}

There is no conflict of interest for the case and informed consent was taken after discussion with patient and attendant about reporting the case for academic interest.

\section{References:}

1. Kumar, V., Abbas, A. K., Fausto, N., Robbins, S. L. \&Cotran, R. S. (2005). Robbins and Cotran pathologic basis of disease (7th ed.). Philadelphia: Elsevier Saunders:pp 272

2. The American Cancer Society medical and editorial content team, (2019, August 14). About melanoma skin cancer. Retrieved from: https://www.cancer.org/cancer/melanoma-skincancer/about/key-statistics.html

3. Krausz MM, Ariel I, Behar AJ. Primary malignant melanoma of the small intestine and the APUD cell concept. J SurgOncol 1978; 10: 283-288, https://doi.org/10.1002/ jso.2930100402, PMid:29168

4. Sato T, Han F, Yamamoto A. The biology and management of uveal melanoma. CurrOncol Rep 2008; 10:431-8. https://doi.org/10.1007/ s11912-008-0066-z, PMid:18706273

5. Lagoudianakis EE, Genetzakis M, Tsekouras DK, Papadima A, Kafiri G, Toutouzas K, Katergiannakis V, Manouras A. Primary gastric melanoma: A case report. World J Gastroenterol 2006; 12(27): 4425-4427, https://doi.org/ 10.3748/wjg.v12.i27.4425, PMid:16865791 PMCid:PMC4087760

6. DasGupta T, Brasfield R. "Metastatic melanoma. A clinicopathological study," Cancer, 1964;17,1323-1339. https://doi.org/ $10.1002 / 1097-0142$ (196410)17: 10 $<1323:: A I D-C N C R 2820171015>3.0 . C O ; 2-N$

7. Hussain MM, Rasul G,Salam GMA. Secondary Melanoma in Gall bladder-- A Case report. J Bangladesh Coll Phys Surg.1992; 10(3): 100-102.

8. Wong K, Serafi SW, Bhatia AS, Irene Ibarra I, Allen EA (2016) Melanoma with gastric metastases, J Community Hosp Intern
MedPerspect. 2016; 6(4), 31972. https://doi.org/10.3402/jchimp.v6.31972 PMid:27609722 PMCid:PMC5016813

9. Faut M, Bisschop $\mathrm{K}$, Jalving $\mathrm{M}$, Been LB, Noordzij W, et al. (2017) Retracted: Diagnosis and Treatment of Intestinal Melanoma Metastases in the Era of Effective Systemic Treatment. Ann Surg. https://doi.org/10.1097/ SLA.0000000000001617, PMid:27140509

10. Patel K, Ward ST, Packer T, Brown S, Marsden J, Thomson M, et al. Malignant melanoma of the gastro-intestinal tract: A case series. Int J Surg. 2014; 12: 523-7. https://doi.org/10.1016/ j.ijsu.2014.02.011, PMid:24576592

11. Farshad S, Keeney S, Halalau A, Ghaith G. A Case of Gastric Metastatic Melanoma 15 Years after the Initial Diagnosis of Cutaneous Melanoma. Case Reports in Gastrointestinal Medicine. 2018 ;2018:7684964. https://doi.org/ 10.1155/2018/7684964, PMid:30151286 PMCid:PMC6087584

12. Wang J, Yang F, Ao WQ, Liu C, Zhang WM, Xu FY. Primary gastric melanoma: A case report with imaging findings and 5-year follow-up. World J Gastroenterol2019; 25(44): 6571-6578, https://doi.org/10.3748/wjg.v25.i44.6571 PMid:31802836 PMCid:PMC6886020

13. Bankar S, Patkar S, Desai S, Shrikhande SV. Unusual presentation of melanoma of unknown primary origin: A case report and review of literature. J Can Res Ther 2015; 11:1025. https://doi.org/10.4103/0973-1482.148680 PMid:26881591

14. Gandini S, Sera F, Cattaruzza MS, Pasquini P, Zanetti R, Masini C, Boyle P, Melchi CF. Meta-analysis of risk factors for cutaneous melanoma: III. Family history, actinic damage and phenotypic factors. European Journal of Cancer. 2005; 41:2040-2059. https://doi.org/ 10.1016/j.ejca.2005.03.034, PMid:16125929 15. Ye Z, Yang Z, Zheng S, Wang W. Robot-assisted laparoscopic surgery for abdominal metastatic melanoma mimicking a gastrointestinal stromal tumor: A case report and review of the literature. Medicine. 2018 Jun; 97(26):e11207. https://doi.org/10.1097/ MD. 0000000000011207, PMid:29952974 PMCid: PMC6039597 
16. Wasif N, Bagaria SP, Ray P, et al. Does metastasectomy improve survival in patients with stage IV melanoma? A cancer registry analysis of outcomes. J SurgOncol 2011;104:111-5. https://doi.org/ 10.1002/ jso.21903, PMid:21381040 PMCid:PMC3199373

17. Ollila DW. Complete metastasectomy in patients with stage IV metastatic melanoma. Lancet Oncol. 2006; 7(11):919-924. https://doi.org/ 10.1016/ S1470-2045(06)70938-X

18. Atkins MB, Hsu J, Lee S, et al. Phase III trial comparing concurrent biochemotherapy with cisplatin, vinblastine, dacarbazine, interleukin-2, and interferon alfa-2b with cisplatin, vinblastine, and dacarbazine alone in patients with metastatic malignant melanoma (E3695): a trial coordinated by the Eastern Cooperative Oncology Group. J ClinOncol. 2008;
26(35):5748-5754. https://doi.org/10.1200/JCO. 2008.17.5448, PMid:19001327 PMCid:PMC2645104

19. CiRen B, Wang X, Long Z. The evaluation of immunotherapy and chemotherapy treatment on melanoma: a network meta-analysis. Oncotarget. 2016;7(49):81493-81511. https://doi.org/ 10.18632/ oncotarget.13277, PMid:27845904 PMCid: PMC5348408

20. Barth A, Wanek LA, Morton DL. Prognostic factors in 1,521 melanoma patients with distant metastases. J Am CollSurg 1995;181:193201.

21. Essner R, Lee JH, Wanek LA, Itakura H, Morton DL. Contemporary surgical treatment of advanced-stage melanoma. Arch Surg. 2004;139(9):961-967, https://doi.org/10.1001/ archsurg.139.9.961, PMid:15381613

\section{Photograph}

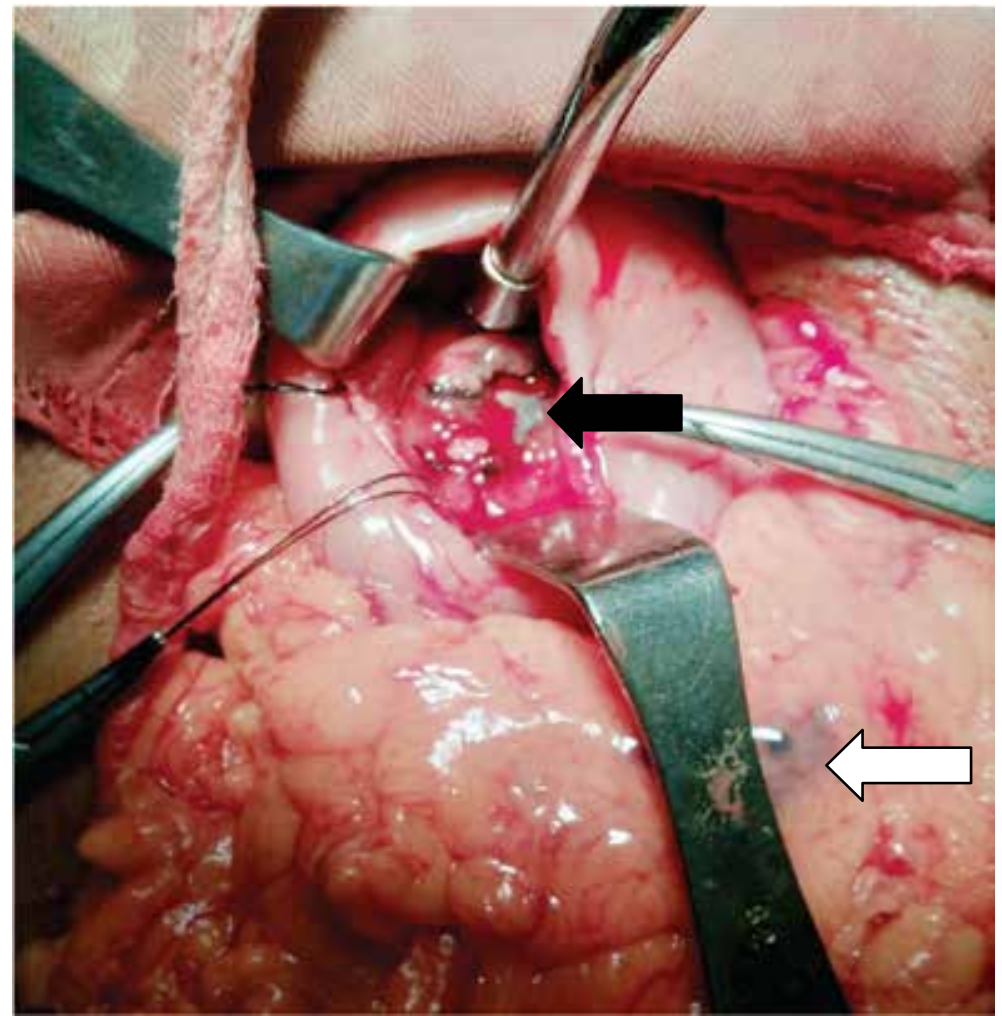

Figure 1: Metastatic gastric melanoma

(The 'black arrow' shows melanoma ulcer crater with blackish color, the 'white arrow' shows black lymph node in omentum) 


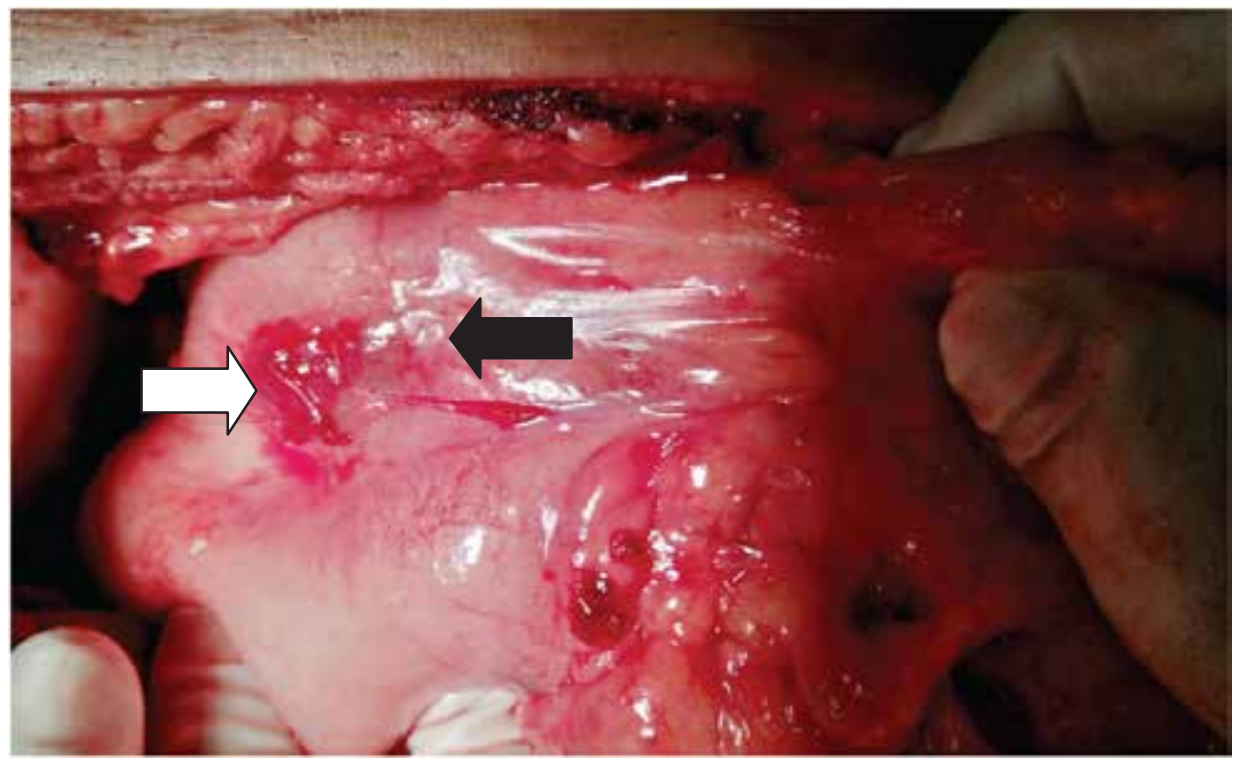

Figure 2: Serosa over greater curvature of stomach

(The 'white arrow' shows induration over greater curvature area, the 'black arrow' shows black color visible from outside of stomach)

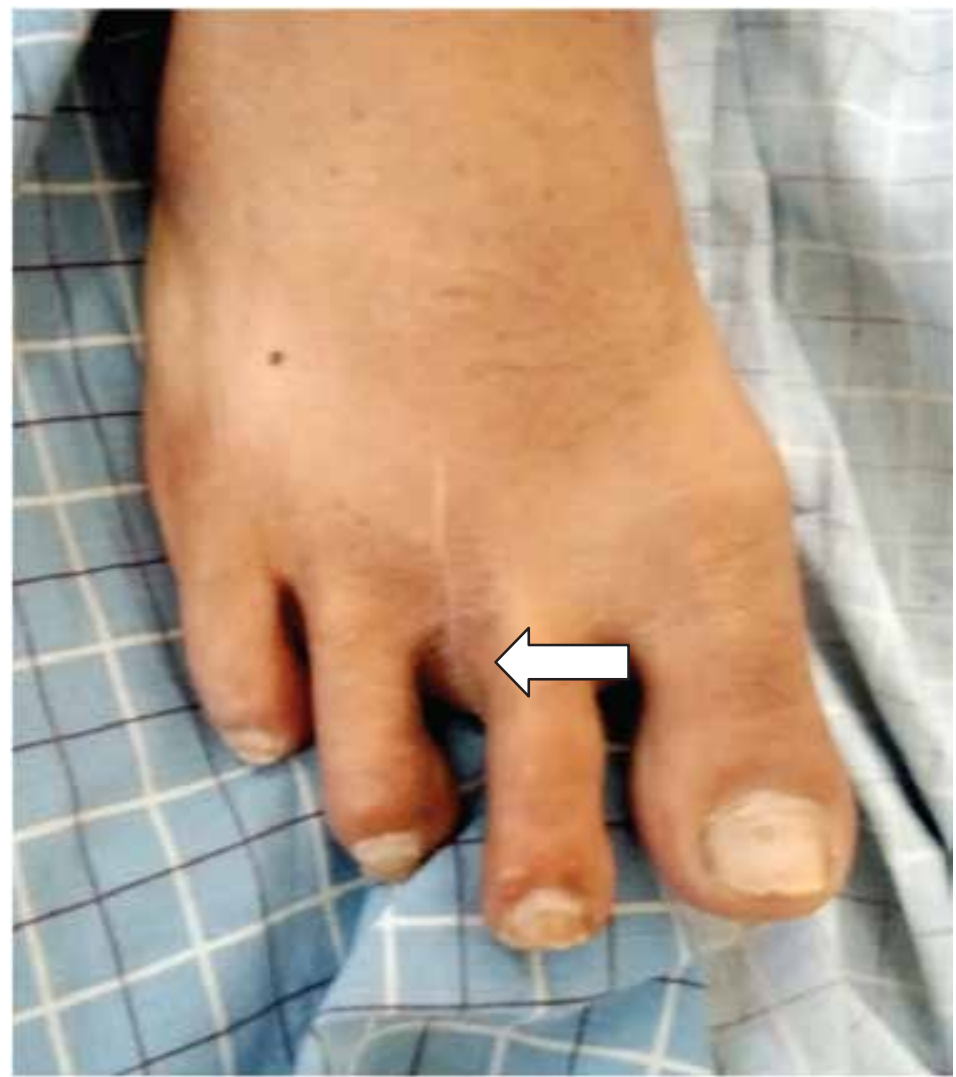

Figure 3: Third toe was amputated three years ago for cutaneous malignant melanoma (The 'white arrow' shows healed scar of amputation site) 


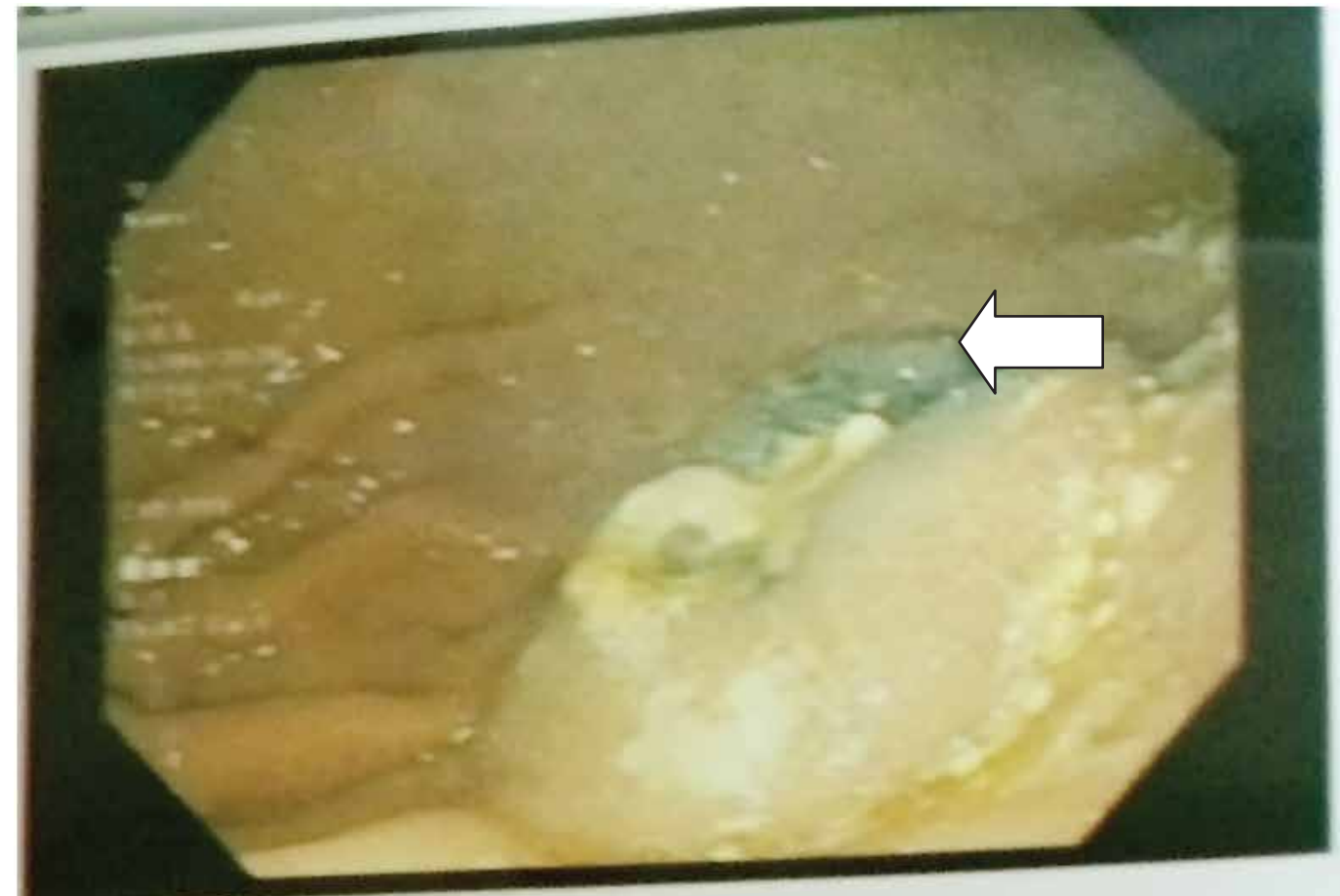

\section{lower gastric body mass}

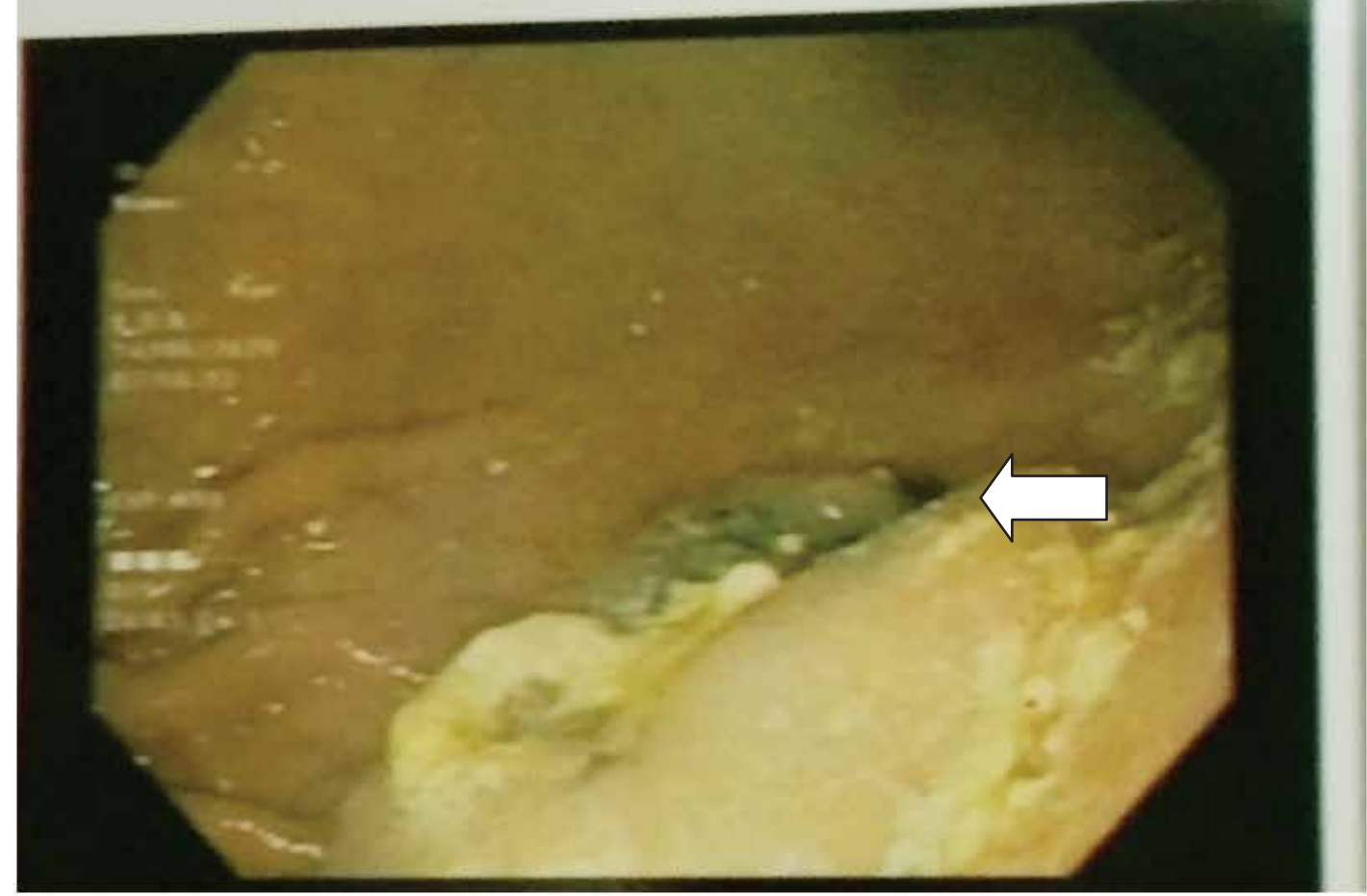

Figure 4: Endoscopic view of gastric melanoma

(Both 'white arrow' shows melanoma ulcer crater with blackish color situated on posterior wall of lower gastric body) 


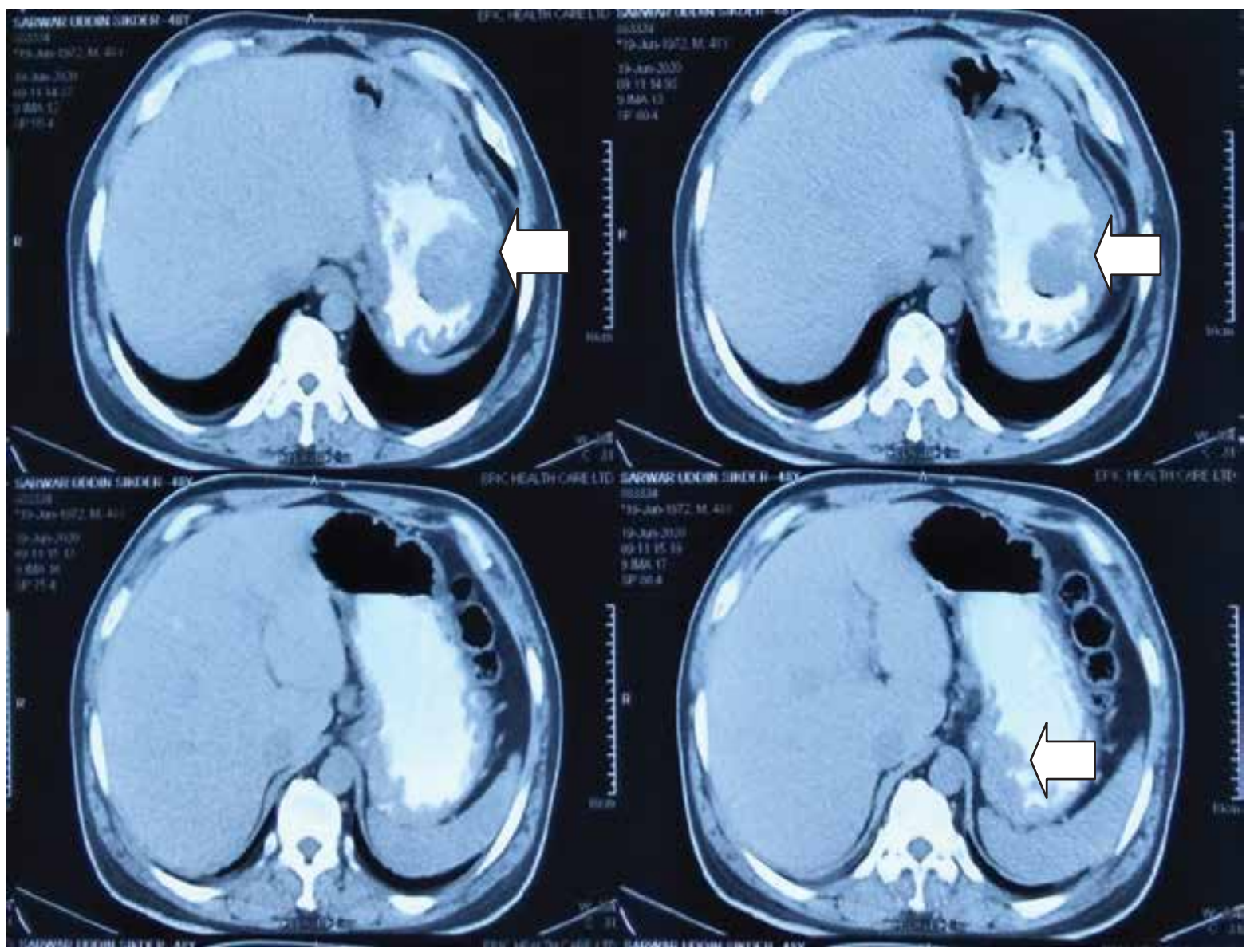

Figure 5: $\mathrm{CT}$ abdomen with contrast showing gastric lesion

('white arrow' shows gastric proliferative lesion with contrast material inside stomach) 